\title{
Kocuria varians infection associated with brain abscess: A case report
}

\author{
Cheng-Yu Tsai', Shou-hsin Su², Yu-Hsin Cheng ${ }^{3}$, Yu-lin Chou'1, Tai-Hsin Tsai and Ann-Shung Lieu*1
}

\begin{abstract}
Background: Kocuria, established by Stackebrandt et al., previously was classified into Micrococcus. Only two species, K. rosea and $K$. kristinae are reported to be associated as pathogenic and found with catheter-related bacteremia and acute cholecystitis.

Case presentation: We herein report the first case of brain abscess caused by Kocuria varians, a gram-positive microorganism, in a 52-year-old man. Hematogenous spread is the probable pathogenesis.

Conclusions: This report presents a case of Kocuria varians brain abscess successfully treated with surgical excision combined with antimicrobial therapy. In addition, Vitek 2 system has been used to identify and differentiate between coagulase-negative staphylococcus.
\end{abstract}

\section{Background}

Kocuria spp. are gram-positive, strictly aerobic microorganisms. Previously they were classified into genus Micrococcus, but have now been removed from Micrococcus based on phylogenetic and chemotaxonomic analysis [1]. This bacterial cluster consists of nine species and is generally considered to be non-pathogenic commensals that colonize the skin, mucosa and oropharynx. However, they can be opportunistic pathogens in immunocompromised patients, though documented cases of infections are rare. We describe a case which presented as a brain abscess caused by Kocuria varians. To our knowledge, this is the first reported case of Kocuria varians associated with brain abscess in the English literature.

\section{Case presentation}

A 52-year-old male, who had a history of diabetes mellitus and hypertension without regular medication control, presented without fever, but with headache and dizziness for 2 months. These headaches were located over the right occipital area without radiation. The headache worsened while thinking or looking at objects for a long time. Also, he did not have any cutaneous/mucosal lesions prior to or during the period in which these head-

* Correspondence: teishin8@hotmail.com

1 Division of Neurosurgery, Department of Surgery, Kaohsiung Medical University Hospital, Kaohsiung, Taiwan

Full list of author information is available at the end of the article aches occurred and he had not received any injections or acupuncture. On hospital admission, he still did not have any fever and physical examination revealed normal findings, but a neurological examination showed left side homonymous hemianopia. Brain computerized tomography (CT) (Fig. 1A) and magnetic resonance (MR) (Fig. 1B) showed a right occipital brain abscess. Systemic third-generation cephalosporins (Ceftazidime) and Metronidaole were initially administered pre-operatively for one day by IV. Then, crainotomy and surgical extirpation were performed promptly after a completed image study identified the abscess. The capsule of the abscess was completely removed during the operation. Pus culture yielded a gram-positive microorganism identified as Kocuria Varians by the Vitek 2 system. Post-operatively, Metronidazole was ceased due to the outcome of an antibiotic susceptibility test report, for 4 weeks Ceftazidime was administered and then shifted to oral form third-generation cephalosporins (Ceftibuten) for 2 weeks. Neurological function was successfully recovered after operation

\section{Microbiological diagnosis}

Culture of brain abscess was performed with sheep blood agar, eosin methylene blue agar and chocolate agar. The plates were incubated at 35 degrees for 48 hours. Anaerobic culture was performed on Bacteroides Bile Esculin agar and incubated at 35 degrees for 48 hours. Gram-pos- 

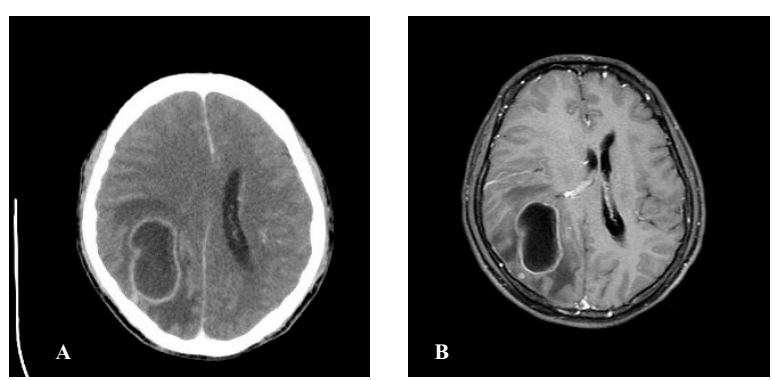

Figure 1 A. Axial brain CT after contrast medium injection showing a ring-enhancement lesion with perifocal edema over right occipital area. B. Axial T1-weighted MR image of the brain after gadolinium injection revealed ring-enhancing cystic lesion with mass effect suggestive of right occipital abscess

itive cocci arranged in tetrads and non-hemolytic, catalase positive, coagulase negative and nonmotile were found. (Fig 2). Anaerobic culture did not find any anaerobic microorganism activity. Identification was performed using the Biome Rieux Vitek 2 system. The isolate was identified as Kocuria varians with very good validity (Fig 3). A useful alternative means of identification, 16s RNA was not performed. The antibiotics sensitivity was performed and sensitive to penicillin, cloxacillin, cefmetazole, clindamycin, Meropenum, penicillin G, Ampicillin/ sulbactan, Piperacillin/tazobactam.

\section{Discussion}

The incidence of brain abscess varies between 1500 to 2500 cases/year in the US, with higher incidences in developing countries [2]. It occurs with the following predisposing sources: 1) contiguous spread; 2) hematogenous spread; 3) head trauma; and 4) neurosurgical procedure. According to the previous literature, the most common modes of direct contiguous spread were from the middle ear, mastoid infections and paranasal sinus [3]. But nowadays, hematogenous spread has become the most common source [2]. The organisms that cause brain abscess are typically bacterial in origin. Streptococcus and staphylococcus are the most frequent organisms and can

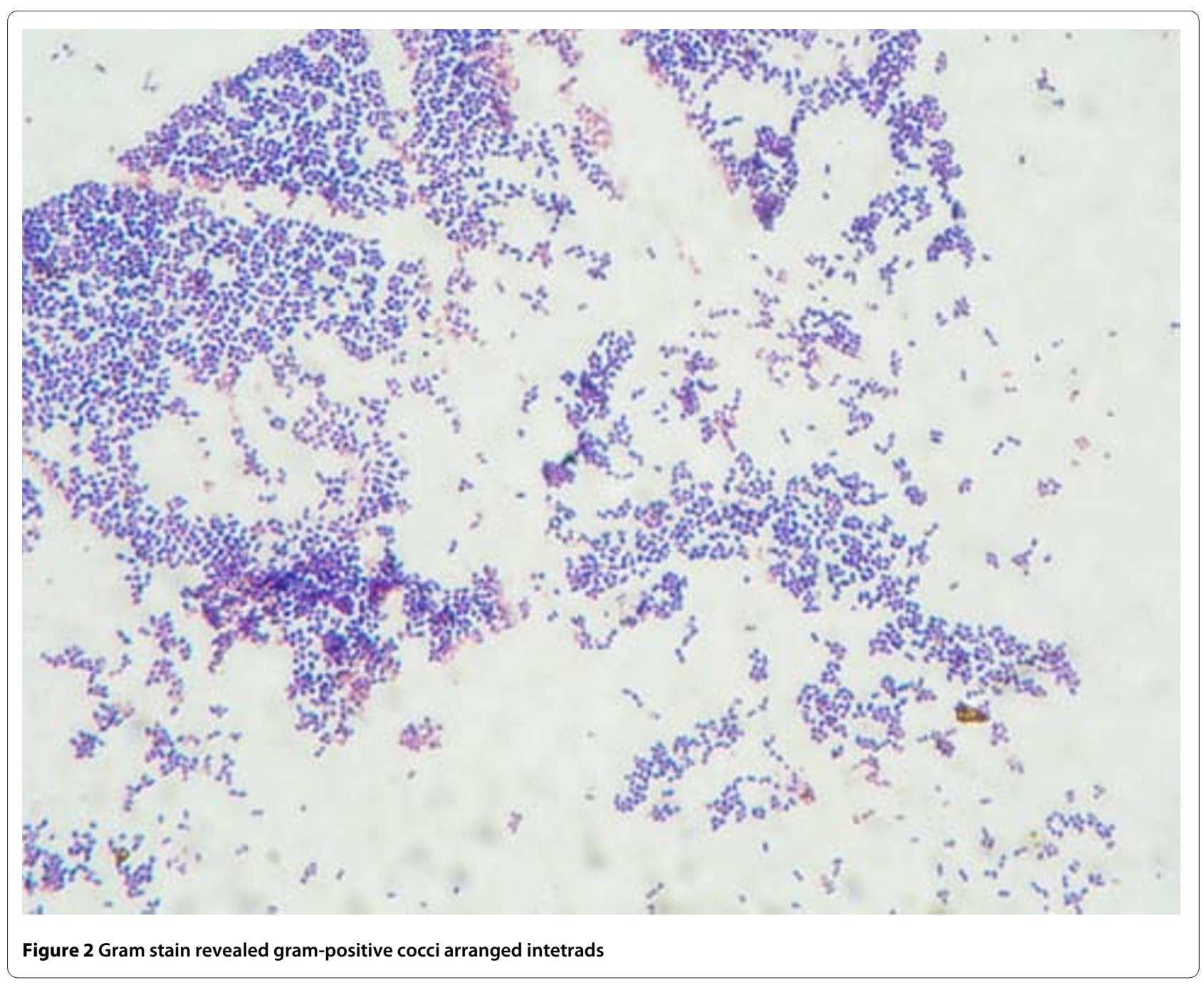




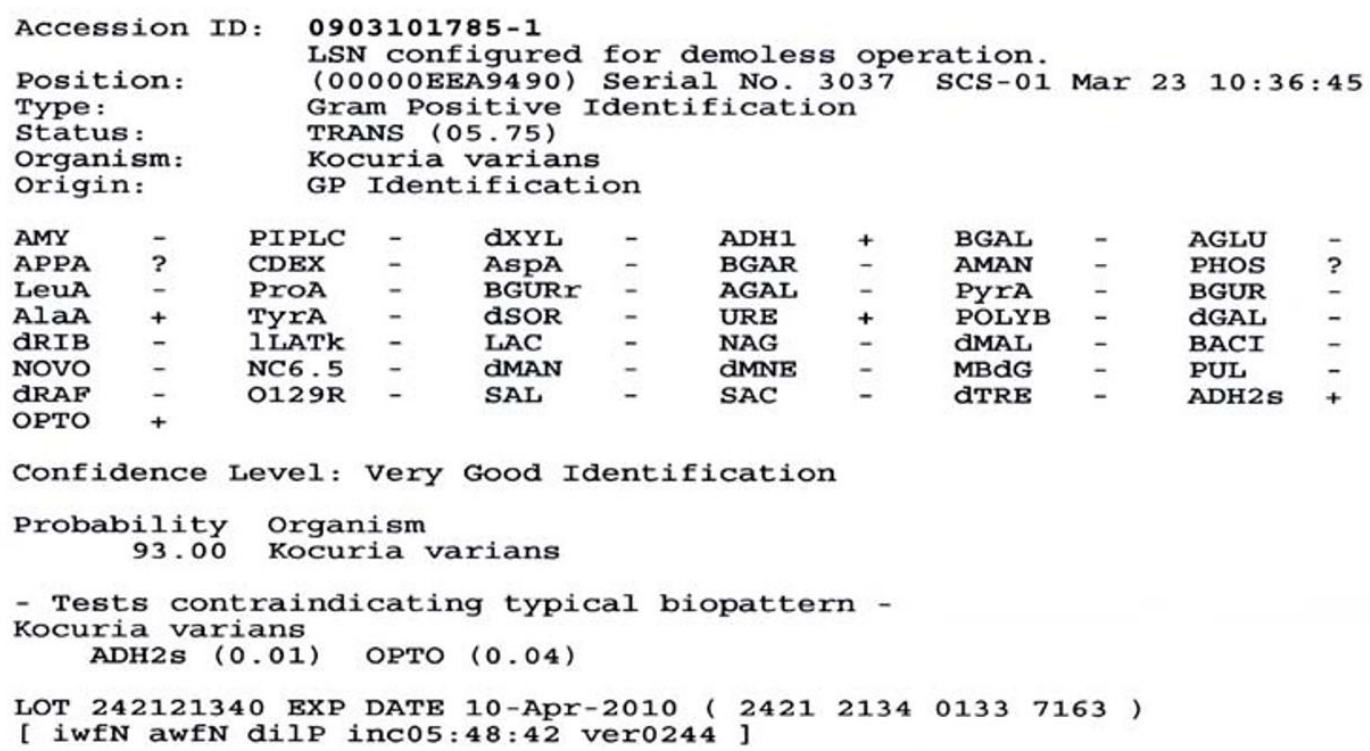

AspA

dSOR

LAC

IMAN

SAL

OPTO +

Confidence Level: Very Good Identification

Probability organism

93.00 Kocuria varians

- Tests contraindicating typical biopattern -

Kocuria varians

ADH2s $(0.01)$ OPTO $(0.04)$

LOT 242121340 EXP DATE 10-Apr-2010 ( 2421213401337163 )

[ iwfN awfN dilp inc05:48:42 ver0244]

Page: 1

Figure 3 Vitek 2 lab reported organism is kocuria varians

be isolated from abscesses of all types and at all sites, whereas Enterobacteriaceae and Bacteroides spp. are isolated from otogenic temporal lobe abscess [4]. In neonates, Proteus and Citrobacter spp. are the most common organisms. Occasionally Mycobacterium tuberculosis as well as fungal infections can act as organisms. Listeria monocytogenes and Burkholderia pseudomallei are rarely reported [4]. Micrococcus spp. associated with brain abscess is mentioned in only two case reports in the English literature [5,8]. In reviewing the English literatures, Kocuria varians related to brain abscess has not yet been documented. Our case is the first reported case in which brain abscess is caused by Kocuria varians.

Kocuria spp. are gram-positive, aerobic microorganisms and found as normal skin flora in humans and other mammals. Infections related to Kocuria spp. are uncommon but can be opportunistic pathogens in immunocompromised patients with underlying diseases. Kocuria spp. was previous classified as Micrococcus spp. Recently, Stackebrant and colleagues made a taxonomic revision of Micrococcus spp. and reclassified it in the new genus Kocuria spp. (Kocuria rosea; K. kristinae; K. varians; K. palustris; and K. rhizophila ap. Nov.) [2,6]. The organism Micrococcus luteus has been reported as a pathogen in meningitis [7], intracranial abscess [8], arthritis [9], pneumonia [10] catheter-related sepsis in patients with hemo- dialysis [11], or leukemia treatment [12]. K. rosea and $K$. kristinae have been reported as pathogens causing catheter-related bacteremia and acute cholecystitis [13-15]. Due to the normal flora of skin or oropharynx and the reported catheter-related bacteremia of other species, the possible predisposing source of Kocuria varians is by hematogenous spread to brain parenchyma.

Due to phenotypic variability, misidentification of coagulase-negative staphylococcus as Kocuria by using standard biochemical analysis is common [16]. Therefore the Vitek 2 system has been used, which is scored as being "very good identification" and is reportedly used in both morphotypes [17]. Also the utilization of genotypic assay, such as 16s RNA is used to confirm species identity [15].

Most strains of Kocuria and Micrococcus were reported to be sensitive to doxycycline, ceftriaxone, cefuroxime, amikacin, and amoxicillin and erythromycin [18], but in consideration of $\mathrm{BBB}$ and penetration of the abscess capsule, third-generation cephalosporins should be used. In our case, surgical excision was performed and then thirdgeneration cephalosporins were administered intravenously for 4 weeks and then shifted to oral form thirdgeneration cephalosporins for 2 weeks as an adequate therapy. 


\section{Conclusion}

Herein we report the first case of Kocuria varians associated with brain abscess in the reviewing the English literature. Because Kocuria spp. is found as normal flora and the hematogenous spread of other related species in reported cases, we supposed Kucuria varians caused brain abscess by hematogenous spreading. Besides Vitek 2 system, 16s RNA may be additionally used to confirm and thus avoid misidentification as coagulase-negative staphylococcus. Surgical excision combined with thirdgeneration cephalosporins could serve as an excellent standard therapy for Kocuria varians brain abscess.

\section{Competing interests}

The authors declare that they have no competing interests.

\section{Authors' contributions}

CT, AL, YC and TT carried out the clinical study of the patient. SS carried out the culture and specific identification of the bacterium. CT drafted the manuscript. All authors read and approved the final manuscript.

\section{Acknowledgements}

Written consent was obtained from the patient for publication of this case report.

\section{Author Details}

'Division of Neurosurgery, Department of Surgery, Kaohsiung Medical University Hospital, Kaohsiung, Taiwan, 2Department of Internal Medicine, Kaohsiung Municipal United Hospital, Kaohsiung, Taiwan and ${ }^{3}$ Department of Nursing, Kaohsiung Medical University Hospital, Kaohsiung, Taiwan

Received: 28 November 2009 Accepted: 27 April 2010 Published: 27 April 2010

\section{References}

1. Stackebrandt E, Koch C, Gvozdiak O, Schumann P: Taxonomic dissection of the genus Micrococcus: Kocuria gen. nov., Nesterenkonia gen. nov., Kytococcus gen. nov., Dermacoccus gen. nov., and Micrococcus Cohn 1872 gen. emend. Int J Syst Bacteriol 1995, 45:682-692.

2. Greenberg MS: Handbook of Neurosurgery. 5th edition. New York: Thieme; 2001:217-223.

3. Jennett B, Miller JD: Infection after depressed fracture of skul implications for management of nonmissile injuries. J Neurosurg 1972 36:333-339.

4. Moorthy RK, Rajshekhar V: Management of brain abscess: an overview. Neurosurg Focus 2008, 24(6):E3.

5. Marchiori $\mathrm{C}$, Tonon $\mathrm{E}$ : Brain abscesses after extracranial infections of the head and neck area. HNO 2003, 51(10):813-22.

6. Màrialigeti K: Kocuria palustris sp. nov. and Kocuria rhizophila sp. nov. isolated from the rhizoplane of the narrow-leaved cattail (Typha angustifolia). Int J Syst Bacterio/ 1999, 49:167-173.

7. Fosse T, Peloux Y, Granthil C, Toga B, Bertrando J, Sethian M: Meningitis due to Micrococcus luteus. Infection 1985, 13:280-281.

8. Selladurai B, Sivakumaran S, Subramanian A, Mohamad AR: Intracranial suppuration caused by Micrococcus luteus. Br J Neurosurg 1993, 7:205-208.

9. Wharton M, Rice JR, McCallum R, Gallis HA: Septic arthritis due to Micrococcus luteus. J Rheumatol 1986, 13:659-660

10. Souhami L, Feld R, TuVnell PG, Fellner T: Micrococcus luteus pneumonia: a case report and review of the literature. Med Pediatr Oncol 1979 7:309-314.

11. Peces R, Gago E, Tejada F, Laures AS, Alvarez-Grande J: Relapsing bacteraemia due to Micrococcus luteus in a haemodialysis patient with Perm-Cath catheter. Nephrol Dial Transplant 1997, 12:2428-2429.

12. Shanks D, Goldwater P, Pena A, Saxon B: Fatal Micrococcus sp. Infection in a child with leukaemia - a cautionary case. Med Pediatr Oncol 2001. 37:553-554.
13. Altuntas F, Yildiz O, Eser B, Gundogan K, Sumerkan B, Cetin M: Catheterrelated bacteremia due to Kocuria rosea in a patient undergoing peripheral blood stem cell transplantation. BMC Infect Dis 2004, 4:62.

14. Basaglia G, Carretto E, Barbarini D, Moras L, Scalone S, Marone P, De Paoli $P$ : Catheter-related bacteremia due to Kocuria kristinae in a patient with ovarian cancer. J Clin Microbiol 2002, 40:311-313.

15. Edmond Ma SK, Chris Wong LP, Kristi Lai TW, Edmond Chan CH, Yam WC, Angus Chan CW: Kocuria kristinae infection associated with acute cholecystitis. BMC Infect Dis 2005, 5:60

16. Ben-Ami R, Navon-Venezia S, Schwartz D, Schlezinger $Y$, Mekuzas $Y$, Carmeli Y: Erroneous reporting of coagulase-negative staphylococci as Kocuria spp. by the Vitek 2 system. J Clin Microbio/ 2005, 43:1448-1450.

17. Ben-Ami R, Navon-Venezia S, Schwartz D, Carmeli Y: Infection of a Ventriculoatrial Shunt with Phenotypically Variable Staphylococcus epidermidis Masquerading as Polymicrobial Bacteremia Due to Various Coagulase- Negative Staphylococci and Kocuria varians. J Clin Microbiol 2003, 41:2444-2447

18. Szczerba I: Susceptibility to antibiotics of bacteria from genera Micrococcus, Kocuria, Nesternkonia, Kytococcus and Dermacoccus. Med Dosw Mikrobiol 2003, 55:75-80.

Pre-publication history

The pre-publication history for this paper can be accessed here: http://www biomedcentral.com/1471-2334/10/102/prepub

doi: $10.1186 / 1471-2334-10-102$

Cite this article as: Tsai et al., Kocuria varians infection associated with brain abscess: A case report BMC Infectious Diseases 2010, 10:102

Submit your next manuscript to BioMed Central and take full advantage of:

- Convenient online submission

- Thorough peer review

- No space constraints or color figure charges

- Immediate publication on acceptance

- Inclusion in PubMed, CAS, Scopus and Google Scholar

- Research which is freely available for redistribution 